\title{
Wavelet Crosstalk Matrix and Its Application to Assessment of Shift-Variant Imaging Systems
}

\author{
Jinyi Qi and Ronald H Huesman
}

\begin{abstract}
The objective assessment of image quality is essential for design of imaging systems. Barrett and Gifford [1] introduced the Fourier crosstalk matrix. Because it is diagonal for continuous linear shift-invariant imaging systems, the Fourier crosstalk matrix is a powerful technique for discrete imaging systems that are close to shift invariant. However, for a system that is intrinsically shiftvariant, Fourier techniques are not particularly effective. Because Fourier bases have no localization property, the shift-variance of the imaging system cannot be shown by the response of individual Fourier bases; rather, it is shown in the correlation between the Fourier coefficients. This makes the analysis and optimization quite difficult. In this paper, we introduce a wavelet crosstalk matrix based on wavelet series expansions. The wavelet crosstalk matrix allows simultaneous study of the imaging system in both the frequency and spatial domains. Hence it is well suited for shiftvariant systems. We compared the wavelet crosstalk matrix with the Fourier crosstalk matrix for several simulated imaging systems, namely the interior and exterior tomography problems, limited angle tomography, and a rectangular geometry positron emission tomograph. The results demonstrate the advantages of the wavelet crosstalk matrix in analyzing shift-variant imaging systems.
\end{abstract}

\section{INTRODUCTION}

The objective assessment of image quality is essential for design of imaging systems. In [1], [2] the concept of Fourier crosstalk matrix was introduced. The imaging system is treated as a continuous-to-discrete mapping and the goal is to recover as many of the Fourier coefficients as possible from the discrete data. The Fourier crosstalk matrix measures how well each Fourier coefficient can be estimated from the data. The diagonal elements of this matrix specify the strength of a Fourier component as reflected in the data, while the off-diagonal elements give the degree of linear dependence of two different components. The design strategy suggested in [1] was to make the crosstalk matrix as nearly diagonal as possible. Recently the Fourier crosstalk matrix was applied to the study of sampling of multi-head coincidence systems [3].

However, making the Fourier crosstalk matrix diagonal is equivalent to requiring the imaging system to be shift-invariant. For imaging systems that are intrinsically shift-variant, such diagonalization is impossible and hence use of the Fourier crosstalk matrix is inappropriate. To deal with the shift-variance of imaging systems, here we propose to use wavelet basis functions rather than the Fourier basis functions in the analysis. The resulting crosstalk matrix is called the wavelet crosstalk matrix. The advantage of using wavelets is that they can capture

\footnotetext{
This work was supported by the U.S. Department of Health and Human Services, the National Institutes of Health under grant P01 HL25840 and by the Director, Office of Science, Office of Biological and Environmental Research, Medical Sciences Division of the US Department of Energy under contract DEAC03-76SF00098

J. Qi and R.H. Huesman are with the Center for Functional Imaging, Lawrence Berkeley National Laboratory, Berkeley, CA 94720 USA.
}

both spatial and frequency information and hence they are a natural choice for analyzing shift-variant systems. We note that wavelets have already used in image reconstruction for tomography, e.g., [4], [5], [6], [7], [8], [9], [10]. Here the wavelet is used to analyze imaging system performance.

Singular value decomposition (SVD) has also been used in analyzing imaging systems (e.g., [11], [12]) and reconstructing images (e.g., [13], [14], [15]). One first chooses a set of basis functions in the object domain (often voxels) and then conducts a SVD on the resulting projection matrix. This is equivalent to the eigenvalue decomposition of the crosstalk matrix. The spectrum of the eigenvalues can be used to evaluate the system performance. One problem with SVD is that when comparing different imaging systems, the set of basis functions (eigenvectors) is often different, which makes the direct comparison of the eigenvalues less meaningful. Another problem is that the eigenvectors can be global functions and hence it is difficult to represent spatial variant responses.

In this paper, we will first review the crosstalk matrix concept and introduce the wavelet crosstalk matrix in Section II. Then in Section III we compare the Fourier crosstalk matrix and wavelet crosstalk matrix for several simulated imaging systems. Finally, the conclusions are drawn in Section IV.

\section{CRosstalk ConcePt}

\section{A. Fourier Crosstalk Matrix}

Barrett et al [1], [2] introduced the Fourier crosstalk matrix in studying cone beam tomography and related it to the figure of merit for task performance. Here we briefly review the concept. Let us consider an object $f(\boldsymbol{x})$ with a finite support $S$, and define an indicator function $S(\boldsymbol{x})$, which is unity when $\boldsymbol{x} \in S$ and zero otherwise. $f(\boldsymbol{x})$ can then be represented exactly by the Fourier series

$$
f(\boldsymbol{x})=\sum_{k=1}^{\infty} F_{k} \Phi_{k}(\boldsymbol{x}),
$$

where

$$
\Phi_{k}(\boldsymbol{x})=\exp \left(2 \pi i \boldsymbol{\rho}_{k} \cdot \boldsymbol{x}\right) S(\boldsymbol{x}) .
$$

Each $\Phi_{k}(\boldsymbol{x})$ is a Fourier basis function truncated by the support region. $\rho_{k}$ is the frequency vector that has the same dimension as $\boldsymbol{x}$ and the '. ' denotes the inner product.

Considering a linear imaging system with $M$ measurements $\left\{y_{m}\right\}_{m=1}^{M}$, we suppose that the expectation of the $m$ th measurement is related to $f(\boldsymbol{x})$ by

$$
\bar{y}_{m}=\int f(\boldsymbol{x}) h_{m}(\boldsymbol{x}) d \boldsymbol{x},
$$

where $h_{m}(\boldsymbol{x})$ is the response function of the $m$ th measurement. Using the above Fourier series expansion, we can express $\bar{y}_{m}$ in 
terms of the Fourier coefficients of $f(\boldsymbol{x})$

$$
\begin{aligned}
\bar{y}_{m} & =\int \sum_{k=1}^{\infty} F_{k} \Phi_{k}(\boldsymbol{x}) h_{m}(\boldsymbol{x}) d \boldsymbol{x} \\
& =\sum_{k=1}^{\infty} F_{k} \int \Phi_{k}(\boldsymbol{x}) h_{m}(\boldsymbol{x}) d \boldsymbol{x} \\
& =\sum_{k=1}^{\infty} F_{k} \Psi_{m k}
\end{aligned}
$$

where $\Psi_{m k}=\int \Phi_{k}(\boldsymbol{x}) h_{m}(\boldsymbol{x}) d \boldsymbol{x}$ is the Fourier transform of the product of the detector response function and the indicator function, with the transform evaluated at frequency $\boldsymbol{\rho}_{k}$.

Equation (6) essentially converts the imaging task from spatial domain into Fourier domain. In order to be able to recover a particular Fourier coefficient $F_{k}$ from the measurements, this Fourier component must make a significant contribution to the data, and this contribution must be distinguishable from the contribution made by other Fourier components.

The crosstalk matrix is a way of quantifying these two problems. The crosstalk matrix $B$ is defined as

$$
B=\Psi^{\dagger} \Psi
$$

where $\Psi^{\dagger}$ is the adjoint (conjugate transpose) of $\Psi$.

The diagonal element $B_{k k}$ measures the strength of the $k$ th Fourier component in the data space; if $B_{k k}$ is zero, the component makes no contribution to the data and cannot be recovered. The off-diagonal element $B_{k k^{\prime}}, k \neq k^{\prime}$ measures the correlation between the contribution of the $k$ th Fourier component and the $k^{\prime}$ th component; the smaller the ratio $\left|B_{k k^{\prime}}\right|^{2} /\left(B_{k k} B_{k^{\prime} k^{\prime}}\right)$, the easier the separation of the two Fourier components. While the full size of $B$ is infinite, any real imaging system can only recover a finite number of Fourier coefficients. Hence we restrict our interest to an $N \times N$ submatrix of $B$, denoted as $B_{N}$. When $f(\boldsymbol{x})$ is band-limited, the submatrix $B_{N}$ is sufficient to characterize the imaging quality.

A general design methodology as discussed in [2] would be to "choose the system geometry in such a way as to minimize the off-diagonal elements of $B_{N}$ and maximize the diagonal elements." Since the Fourier crosstalk matrix is diagonal for continuous linear shift-invariant systems, minimizing the offdiagonal elements of $B_{N}$ is equivalent to make the system shiftinvariant. Thus this method is useful for designing imaging systems that are close to shift-invariant. If, however, the imaging system is intrinsically shift-variant, and the designer has no intention to make it shift-invariant, minimizing the off-diagonal elements of $B_{N}$ would not be appropriate since the off-diagonal elements can be caused by the shift-variant response. Moreover, because Fourier basis functions are global functions, it is difficult to deduce the spatially variant response of a shift-variant system. Hence, the Fourier crosstalk matrix is not effective for analyzing shift-variant systems.

\section{B. Wavelet Crosstalk Matrix}

To assess the image quality of shift-variant imaging systems, we propose the wavelet series expansion instead of the Fourier series expansion. The wavelet series expansion preserves both spatial and frequency information, and thus is more suitable for analyzing shift-variant imaging systems.

Using wavelets, $f(\boldsymbol{x})$ can be represented by [16]

$$
f(\boldsymbol{x})=\sum_{l=-\infty}^{\infty} \sum_{n=-\infty}^{\infty} d_{n}^{(l)} \psi_{l, n}(\boldsymbol{x}),
$$

where $\psi_{l, n}(\boldsymbol{x})$ is the $n$th wavelet at the $l$ th scale and $d_{n}^{(l)}$ is the wavelet coefficient. When $f(\boldsymbol{x})$ is band-limited with a finite support, the infinite sums can be approximated by finite sums. A single index $k$ will also be used in place of the double indices $(l, n)$ by defining a one-to-one mapping between $k$ and $(l, n)$. Using this wavelet series expansion, the data of the imaging system can be expressed using wavelet coefficients

$$
\begin{aligned}
\bar{y}_{m} & =\int \sum_{k=-\infty}^{\infty} d_{k} \psi_{k}(\boldsymbol{x}) h_{m}(\boldsymbol{x}) d \boldsymbol{x} \\
& =\sum_{k=-\infty}^{\infty} d_{k} \int \psi_{k}(\boldsymbol{x}) h_{m}(\boldsymbol{x}) d \boldsymbol{x} \\
& =\sum_{k} d_{k} W_{m k},
\end{aligned}
$$

where $W_{m k}=\int_{S} \psi_{k}(\boldsymbol{x}) h_{m}(\boldsymbol{x}) d \boldsymbol{x}$.

We can then obtain the wavelet crosstalk matrix

$$
C=W^{T} W
$$

The diagonal element $C_{k k}$ measures the strength of the contribution of the $k$ th wavelet to the data, and the off-diagonal element $C_{k k^{\prime}}, k \neq k^{\prime}$ measures the correlation between the contribution of the two wavelets. The advantage of the wavelet crosstalk matrix is that it can simultaneously capture both spatial and frequency information. Thus it is more efficient than the Fourier crosstalk matrix in analyzing shift-variant imaging systems.

\section{Generalization of the Crosstalk Concept and Relation to Least Squares Estimate}

The crosstalk concept can be generalized to any basis functions that we would use to represent the object $f(\boldsymbol{x})$. One of the most widely used bases is the cubic voxel, i.e.,

$$
f(\boldsymbol{x})=\sum_{k=1}^{\infty} v_{k} I_{k}(\boldsymbol{x}),
$$

where each basis $I_{k}(\boldsymbol{x})$ is an indicator function on a cubic region centered at one of the image sampling points in a 3D lattice. The single index $k$ represents the lexicographically ordered points on the lattice. The expectation of data can be expressed by

$$
y_{m}=\sum_{k=1}^{\infty} v_{k} P_{m k},
$$

where $P_{m k}=\int_{I_{k}} h_{m}(\boldsymbol{x}) d \boldsymbol{x}$.

We then obtain the voxel crosstalk matrix

$$
H=P^{T} P
$$


Note that the least squares estimate of $v_{k}$ from data $\boldsymbol{y}$ is

$$
\hat{\boldsymbol{v}}^{L S}=\left[P^{T} P\right]^{-1} P^{T} \boldsymbol{y}=H^{-1} P^{T} \boldsymbol{y} .
$$

This indicates that the crosstalk matrix is closely related to the least squares solution. This is also true for any other basis functions.

The least square solution is optimum for white Gaussian noise. When noise from different detectors is different or correlated, a weighted least squares solution is more statistically efficient.

$$
\hat{\boldsymbol{v}}^{W L S}=\left[P^{T} \Sigma^{-1} P\right]^{-1} P^{T} \Sigma^{-1} \boldsymbol{y},
$$

where $\Sigma$ is the noise covariance matrix.

With analogy to (11), we can define a weighted crosstalk matrix

$$
H_{w}=P^{T} \Sigma^{-1} P .
$$

Similarly, this weighted crosstalk matrix can be used to evaluate imaging systems with colored noise. We note that the weighted crosstalk matrix is essentially the same as the Fisher information matrix for Gaussian noise and hence it can be directly related to task specific figures of merit.

\section{Simulation Results}

We conduct computer simulations to compare the wavelet crosstalk matrix with the Fourier crosstalk matrix for different imaging systems. The wavelet that we use here is the Haar wavelet [16]. For all crosstalk matrices, we use $32 \times 32$ basis functions. In order to model the exact response function in the simulated imaging system, the response function is first computed on a $256 \times 256$ fine pixel grid. The eight-fold oversampling is to minimize the discretization effect. The response functions of the Fourier and wavelet bases are then calculated using the fast Fourier transform and wavelet transform, respectively, and only the $32 \times 32$ basis functions with the lowest frequency are kept. For Fourier basis functions with line integral model, the results of this method agree well with that of the continuous model used in [2], [3].

\section{A. Interior Problem and Exterior Problem}

We first simulate the classic interior and exterior problems [17, p.158]. We choose an example in two-dimensional Radon tomography with parallel projections for which the original full projection has 40 view angles and 32 lines of response (LORs) per view with a sampling distance of $1 \mathrm{~mm}$. The interior problem is simulated by only keeping 11 LORs at the center of each view angle and the exterior problem is simulated by removing the 11 LORs at the center in each view angle. The fields of view of the two problems are both $32 \times 32 \mathrm{~mm}^{2}$. We compute the pixel, Fourier, and wavelet crosstalk matrices for the two simulated problems. The diagonal elements are shown in Figs. 1 and the absolute correlation coefficients are shown in Fig. 2.

For the interior problem, the diagonal elements of the pixel crosstalk matrix show large contributions from pixels within the interior region in the field of view. For the exterior problem, the contributions only come from pixels in the exterior region. However, the diagonals of the two Fourier crosstalk matrices are quite similar, showing each Fourier basis makes similar contribution to both problems. While there is slight difference in the

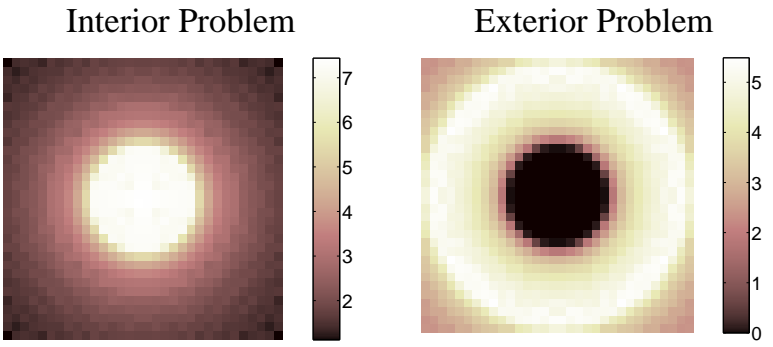

(a) Pixels

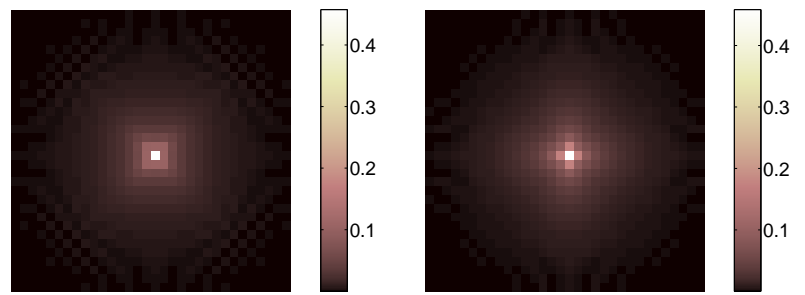

(b) Fourier

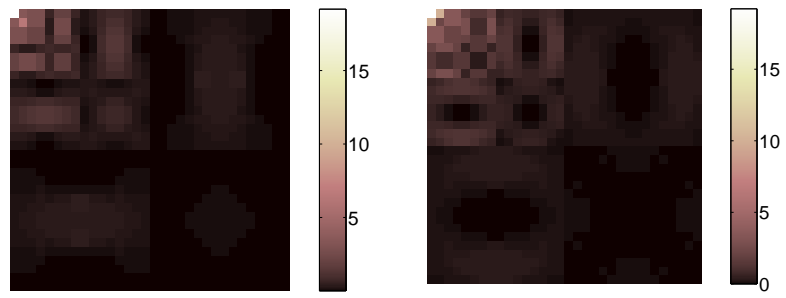

(c) Wavelets

Fig. 1. The diagonal elements of (a) the pixel crosstalk matrix, (b) the Fourier crosstalk matrix, and (c) the wavelet crosstalk matrix for the interior problem and the exterior problem. The DC component is at the center in the Fourier image, and is at the upper left corner in the wavelet image.

off-diagonals of the Fourier crosstalk matrices for the two problems as shown in the correlation images (Fig. 2), it is rather difficult to distinguish the features of the two problems using the Fourier crosstalk matrix. In comparison, the wavelet crosstalk matrices clearly capture the distinct features of the two problems by preserving both spatial and frequency information, i.e., combination of the pixel crosstalk matrix and Fourier crosstalk matrix. It shows that the wavelets corresponding to the interior region make the major contributions to data in the interior problem and the contributions from the wavelets in the exterior region depend on the spatial and frequency locations of the wavelets. For example, at the top and bottom regions, only vertical wavelets have contributions, while at the left and right, only horizontal wavelets have contributions. For the exterior problem only wavelets of the exterior region have contributions and again it depends on the spatial and frequency locations of the wavelets.

\section{B. Dual-planar PET}

Here we consider a dual-planar design of a positron emission tomography (PET) system for imaging breast (Fig. 3a). This system has two parallel detector heads and each has 32 detectors. All the lines of response are collected. With the finite detector heads, the view angles are limited, and there are varying numbers of LORs in each view. In Fig. 3 we show the diagonals of the pixel, Fourier, and wavelet crosstalk matrices. In Fig. 4, 
Interior Problem
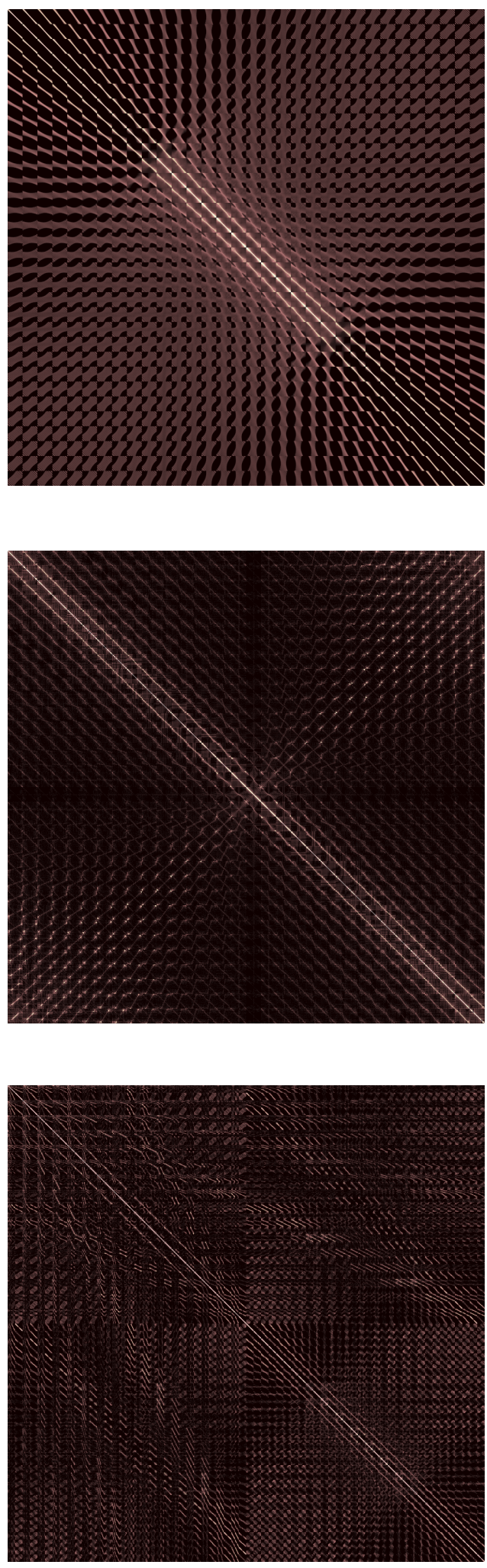

Exterior Problem
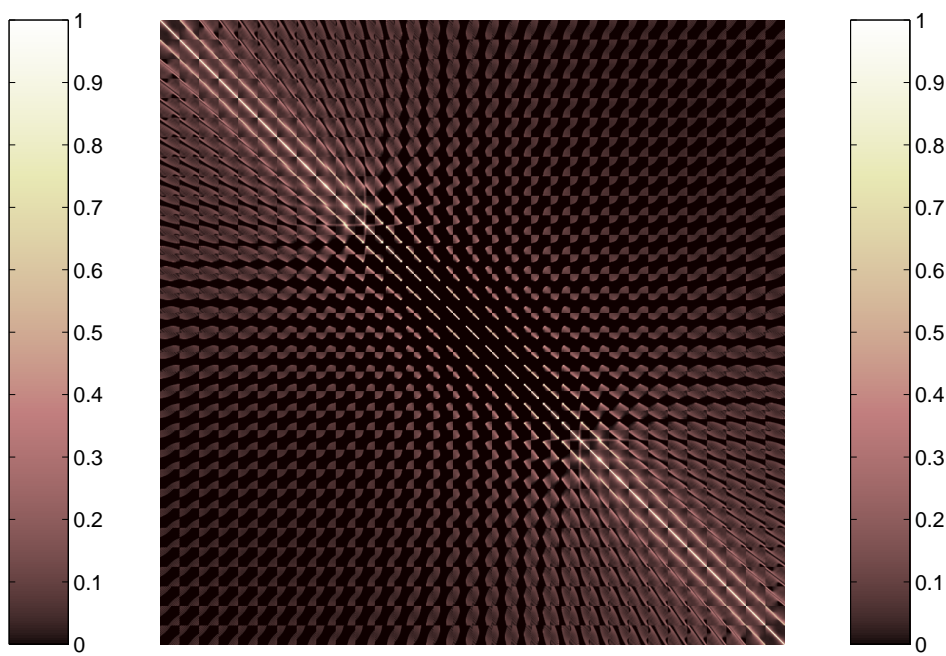

(a) Pixels
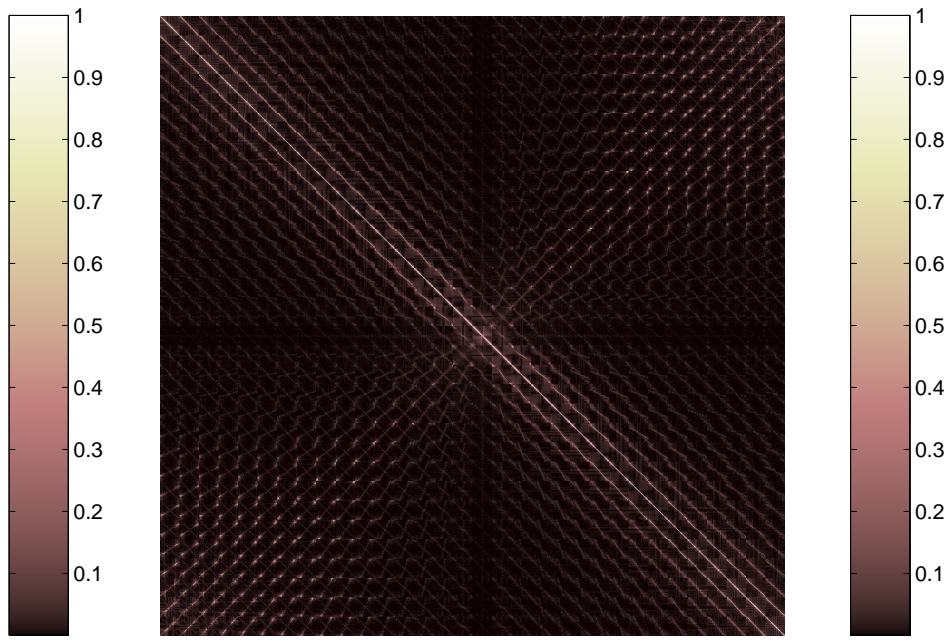

(b) Fourier

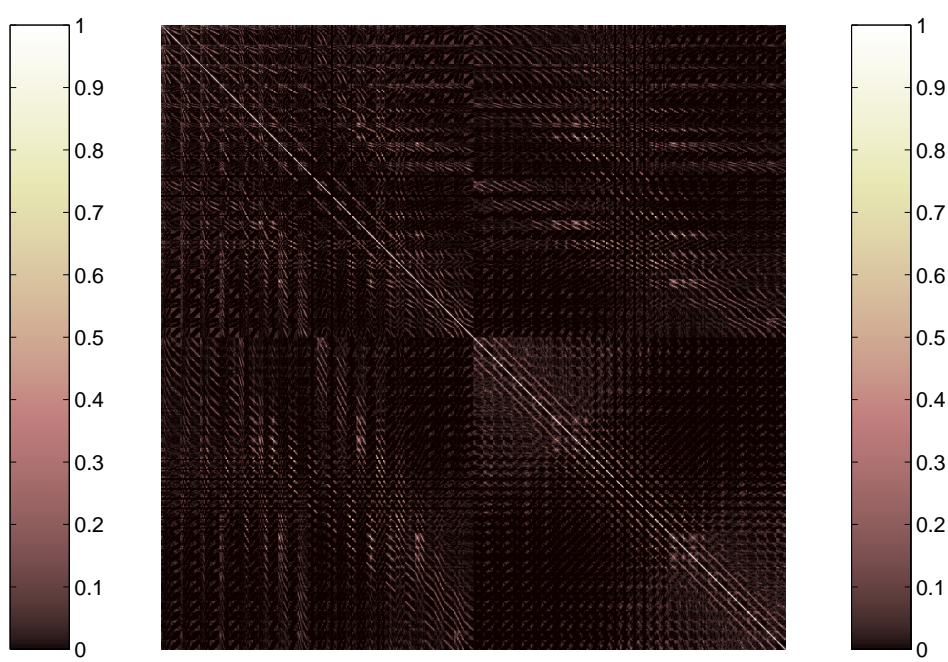

(c) Wavelets

Fig. 2. The absolute value of the correlations between the (a) pixel bases, (b) Fourier bases, and (c) wavelet bases for the interior problem and the exterior problem. The off-diagonals have been divided by the square root of the product of the corresponding diagonals and the diagonals have been normalized to unity. 


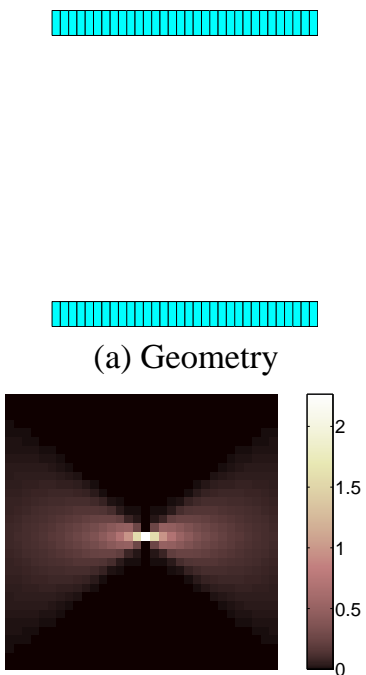

(c) Fourier

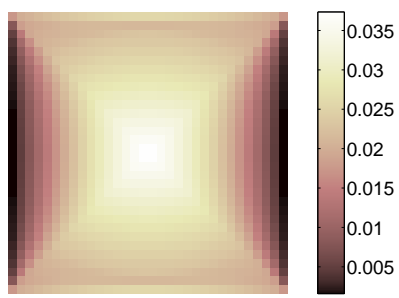

(b) Pixels

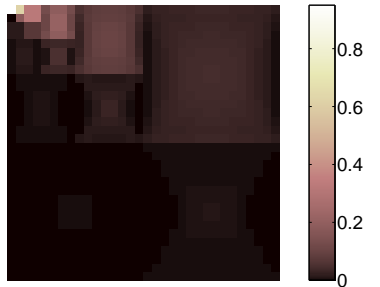

(d) Wavelets

Fig. 3. (a) The dual-planar system. The diagonal elements of (b) the pixel crosstalk matrix, (c) the Fourier crosstalk matrix, and (d) the wavelet crosstalk matrix. The DC component is at the center in the Fourier image, and is at the upper left corner in the wavelet image.

we show the correlation images of the Fourier crosstalk matrix and the wavelet crosstalk matrix. Again, it is rather difficult to see the spatially variant response of the imaging system from the Fourier crosstalk matrix as such variation is woven into the correlations between Fourier coefficients. In contrast, the diagonal of the wavelet crosstalk matrix captures both the spatial and frequency response of the dual-planar system. The strong correlations between wavelet coefficients are largely due to the fact that such limited angle tomography is very ill-posed.

\section{Rectangular PET}

The last imaging system that we simulate is a rectangular PET system (Fig. 5a). This design is also being pursued for breast imaging [18]. Because of the solid angle effect, the short LORs (near the corners) have higher sensitivity than the long LORs. As a result, the rectangular geometry causes highly shift-variant sensitivity as shown by the diagonals of the pixel crosstalk matrix (Fig. 5b). The calculated wavelet crosstalk matrix and Fourier crosstalk matrix for this system are shown in Fig. 6 and Fig. 7.

In this case, the spatially variant response causes strong correlations between different Fourier components (Fig. 7a). By just looking at the Fourier crosstalk matrix, one might doubt that this rectangular PET could recover any Fourier component. However, we know that this system has complete sampling and the result should not be so bad. This is clearly shown by the wavelet crosstalk matrix. The energy is more concentrated around diagonal elements and the correlations between different wavelets are relatively small. The wavelet crosstalk matrix also shows that the rectangular PET has much higher sensitivity near the corners (Fig. 6b). This clearly demonstrates the advantage of the wavelet crosstalk matrix.

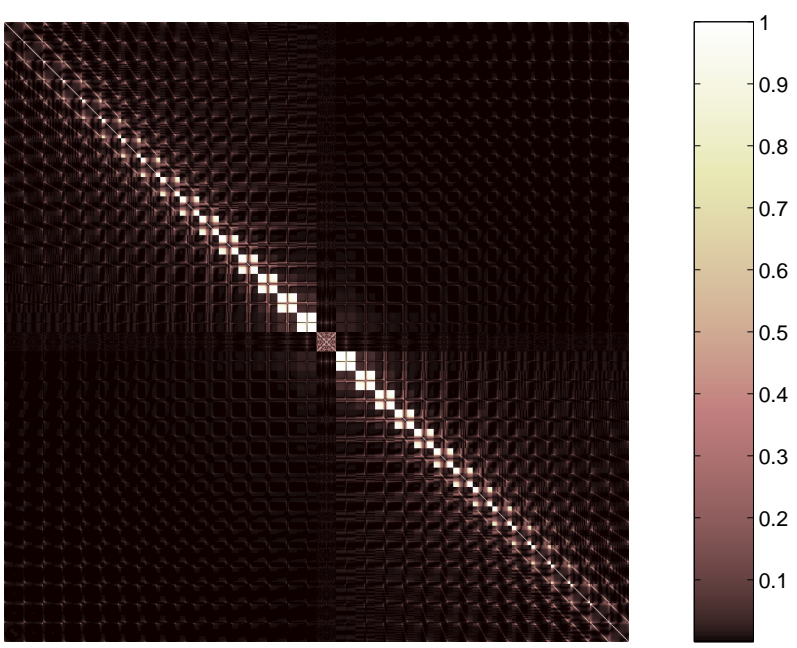

(a) Fourier

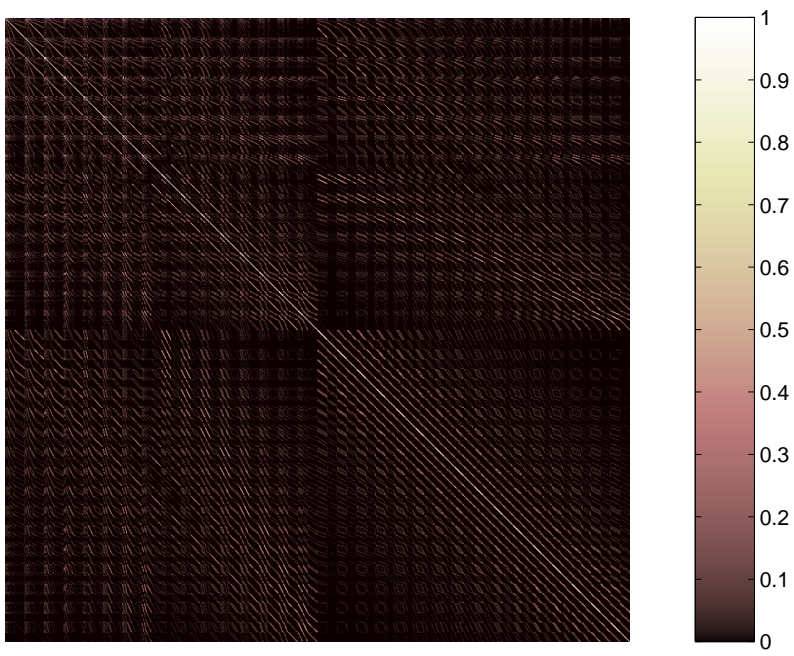

(b) Wavelets

Fig. 4. The correlation images of (a) the Fourier crosstalk matrix and (b) the wavelet crosstalk matrix for the rectangular PET. The off-diagonals have been divided by the square root of the product of the corresponding diagonals and the diagonals have been normalized to unity.

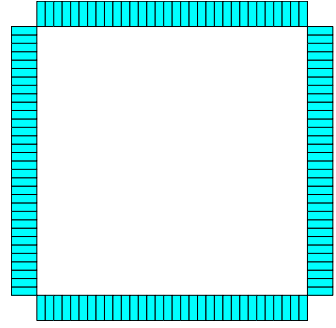

(a) Geometry

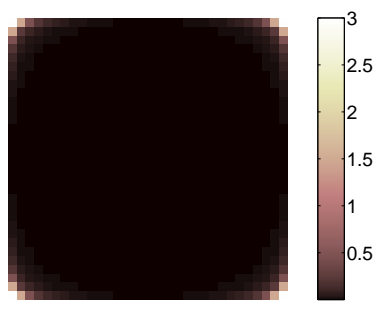

(b) Pixels
Fig. 5. (a) A rectangular PET system with 32 detectors on each side. The field of view is the whole square enclosed by the detectors. (b) The diagonals of the pixel crosstalk matrix (the maximum intensity has been truncated to shown more details). 


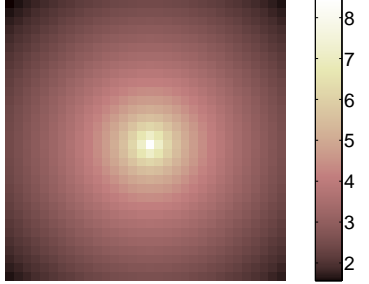

(a) Fourier

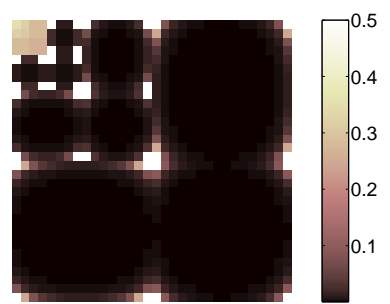

(b) Wavelets
Fig. 6. The diagonal elements of (a) the Fourier crosstalk matrix and (b) the wavelet crosstalk matrix for the rectangular PET. The DC component is at the center in the Fourier image, and at the lower right corner in the wavelet image. The intensity in the wavelet image is truncated.

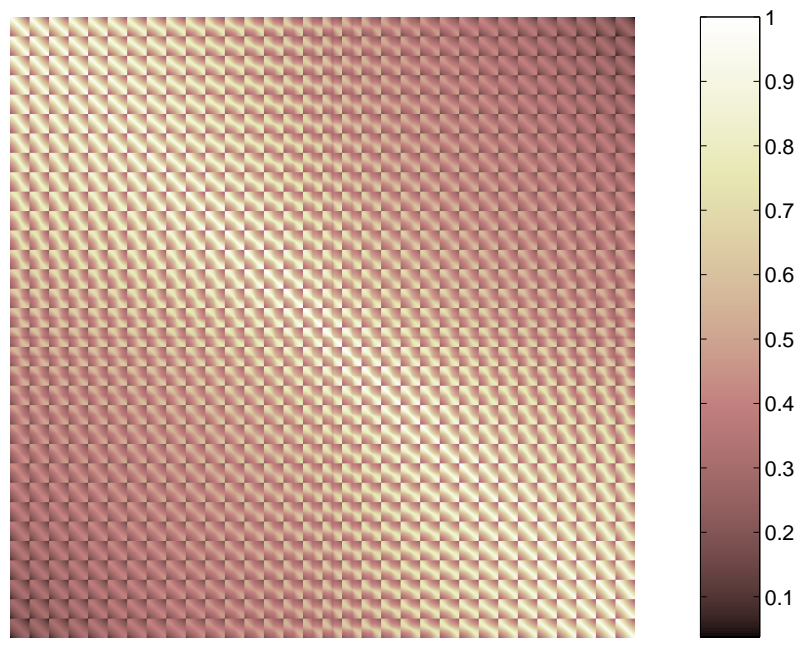

(a) Fourier

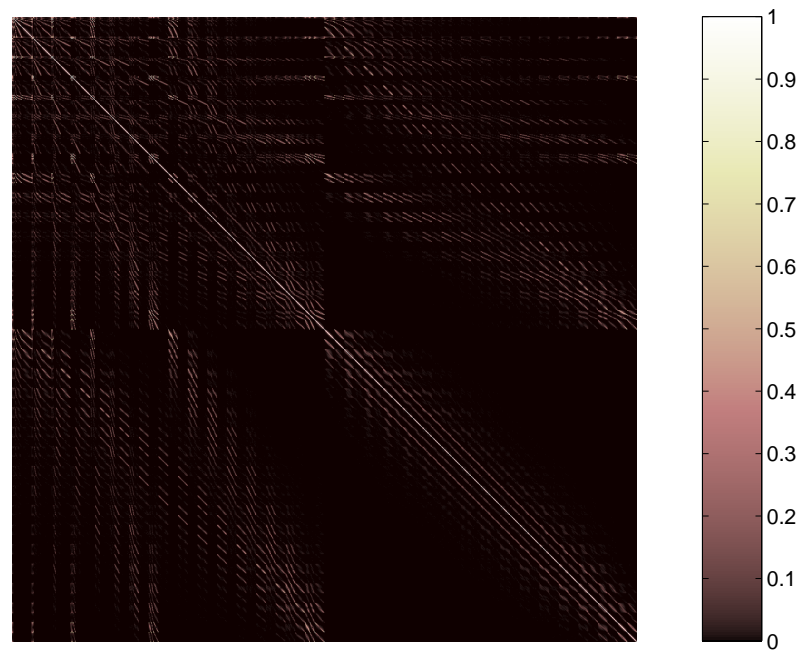

(b) Wavelets

Fig. 7. The correlation images of (a) the Fourier crosstalk matrix and (b) the wavelet crosstalk matrix for the rectangular PET. The off-diagonals have been divided by the square root of the product of the corresponding diagonals and the diagonals have been normalized to unity.

\section{CONCLUSION AND DISCUSSION}

We have introduced the wavelet crosstalk matrix based on the wavelet series expansion of the object function. In comparison to the Fourier crosstalk matrix, the wavelet crosstalk matrix can simultaneously capture both spatial and frequency information and hence is more efficient for the analysis of shift-variant imaging systems.

We have also linked the crosstalk matrix with least squared estimation and have introduced the weighted crosstalk matrix for weighted least squared estimation. We pointed out the equivalence between the (weighted) crosstalk matrix and the Fisher information matrix for Gaussian noise. Thus, the crosstalk matrix can be directly related to task specific figures of merit.

We have demonstrated the advantages of the wavelet crosstalk matrix using computer simulations. Future work will include applying the wavelet crosstalk matrix to analysis of imaging systems with complex detector geometry and/or motion.

\section{REFERENCES}

[1] H. H. Barrett and H. C. Gifford, "Cone-beam tomgraphy with discrete data sets," Physics in Medicine and Biology, vol. 39, pp. 451-476, 1994.

[2] H. H. Barrett, J. L. Denny, R. F. Wagner, and K. J. Myers, "Objective assessment of image quality. II. Fisher information, Fourier crosstalk, and figures of merit for task performance," Journal of Optical Society of America A, vol. 12, pp. 834-852, 1995.

[3] R. Stodilka, E. Soares, and S. Glick, "Characterization of tomographic sampling in hybrid-PET using the Fourier crosstalk matrix," in Proceedings of IEEE Nuclear Science Symposium and Medical Imaging Conference, p. 16/39, Lyon, France, 2000.

[4] Y. Choi, J. Y. Koo, and N. Y. Lee, "Image reconstruction using the wavelet transform for positron emission tomography," IEEE Transactions on Medical Imaging, vol. 20, pp. 1188-1193, 2001.

[5] S. Y. Zhao and G. Wang, "Feldkamp-type cone-beam tomography in the wavelet framework," IEEE Transactions on Medical Imaging, vol. 19, pp. 922-929, 2000.

[6] A. Raheja and A. P. Dhawan, "Wavelet based multiresolution expectation maximization image reconstruction algorithm for positron emission tomography," Computerized Medical Imaging and Graphics, vol. 24, pp. 359-376, 2000.

[7] S. Bonnet, F. Peyrin, F. Turjman, and R. Prost, "Tomographic reconstruction using nonseparable wavelets," IEEE Transactions on Image Processing, vol. 9, pp. 1445-1450, 2000.

[8] M. Bhatia, W. C. Karl, and A. S. Willsky, "A wavelet-based method for multiscale tomographic reconstruction," IEEE Transactions on Medical Imaging, vol. 15, pp. 92-101, 1996.

[9] A. H. Delaney and Y. Bresler, "Multiresolution tomographic reconstruction using wavelets," IEEE Transactions on Image Processing, vol. 4, pp. 799-813, 1995.

[10] M. Unser and A. Aldroubi, "A review of wavelets in biomedical applications," Proceedings of the IEEE, vol. 84, pp. 626-638, 1996.

[11] G. L. Zeng and G. T. Gullberg, "An SVD study of truncated transmission data in SPECT," IEEE Transactions on Nuclear Science, vol. 44, pp. 10711, 1997.

[12] G. Zeng, G. Gullberg, and S. Foresti, "Eigen analysis of cone-beam scanning geometries," in International Meeting on Fully Three Dimensional Image Reconstruction in Radiology and Nuclear Medicine, pp. 261-5, Aix-les-Bain, France, 1995

[13] G. T. Gullberg, Y.-L. Hsieh, and G. L. Zeng, "An SVD reconstruction algorithm using a natural pixel representation of the attenuated radon transform," IEEE Transactions on Nuclear Science, vol. 43, pp. 295-303, 1996.

[14] M. F. Smith, "Generalized matrix inverse reconstruction for SPECT using a weighted singular value spectrum," IEEE Transactions on Nuclear Science, vol. 43, pp. 2008-2017, 1996.

[15] V. V. Selivanov and R. Lecomte, "Fast PET image reconstruction based on SVD decomposition of the system matrix," IEEE Transactions on Nuclear Science, vol. 48, pp. 761-7, 2001.

[16] M. Vetterli and J. Kovacevic, Wavelets and Subband Coding, Prentice Hall PTR, 1995.

[17] F. Natterer, The Mathematics of Computerized Tomography, John Wiley and Sons, 1986. 
[18] P. Virador, W. Moses, and R. Huesman, "Reconstruction in PET cameras with irregular sampling and depth of interaction capability," IEEE Transactions on Nuclear Science, vol. 45, pp. 1225-1230, 1998. 